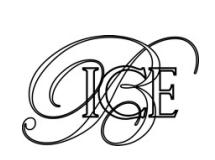

Eduardo Aguilar García *

Carolina Mateos Bustamante*

\title{
PREMIO NOBEL DE ECONOMÍA 2015 Angus Deaton
}

El 12 de octubre de 2015, la Real Academia Sueca otorgó el premio Sveriges Riksbank de Ciencias Económicas en Memoria de Alfred Nobel a Angus Deaton por sus análisis sobre el consumo, la pobreza y el bienestar. El profesor Deaton es una de las figuras más admiradas de la ciencia económica cuyas aportaciones han contribuido decisivamente al progreso de la economía. Los hitos más importantes de su carrera académica se resumen en tres grandes ejes: la modelización y estimación del Sistema Casi Ideal de Demanda, los estudios sobre la teoría del consumo intertemporal y el desarrollo de encuestas de hogares para la medición de la pobreza y los niveles de vida en países en vías de desarrollo. Sin embargo, su legado se extiende por todas las ramas de la ciencia económica y le sitúa como uno de los economistas más influyentes de la actualidad.

Palabras clave: pensamiento económico, teoría del consumo, pobreza, bienestar, desigualdad. Clasificación JEL: B31, C32, D12.

\section{Introducción}

Históricamente, la concesión del premio Sveriges Riksbank de Ciencias Económicas en Memoria de Alfred Nobel, oficiosamente conocido como el Premio Nobel de Economía, no ha estado exenta de polémica. Pese a que la lista de ganadores del prestigioso galardón es una selección de los economistas con mayor influencia en la teoría económica moderna, acertar el vencedor de cada edición se ha convertido en una tarea casi imposible. Los premiados de las pasadas ediciones son, sin lugar a duda, grandes figuras de la disciplina con un marcado perfil técnico, pero no economistas mediáticos. Tirole fue elegido en 2014 por sus estudios sobre regulación de monopolios y Shiller,

\footnotetext{
* Técnicos Comerciales y Economistas del Estado.

Versión de noviembre de 2015.
}

Hansen y Fama fueron premiados en 2013 por sus investigaciones sobre el proceso de formación de precios de activos. Otro buen ejemplo son Roth y Shapley, galardonados en 2012 por sus aportaciones al conocimiento del diseño de los mercados a pesar de que Shapley no se definía como economista. En 2005, Aumann, matemático experto en teoría de juegos, reconocía que hasta hacía poco no sabía lo que era la inflación y Ostrom, premiada en 2009 , era politóloga.

Sin embargo, con la concesión del Premio Nobel de Economía de 2015 a Angus Deaton por sus análisis sobre consumo, pobreza y bienestar, la Real Academia Sueca galardona a una de las figuras más admiradas, tanto por neoclásicos como por keynesianos, de la ciencia económica. Es además una eminencia en el estudio de la pobreza y la desigualdad, campos que desde hace unos años han ido $D$ 
adquiriendo mayor importancia dentro de la profesión gracias a los estudios de Bhagwati, Milanovic, Duflo, Banerjee o el propio Deaton.

Su biografía y sus aportaciones a la microeconomía, la macroeconomía y la economía del desarrollo retratan a un economista que ha revolucionado en muchos sentidos la forma que tenemos de entender la relación entre microeconomía y macroeconomía, entre teoría y evidencia empírica, así como la forma de analizar la pobreza y el desarrollo, la renta y la distribución. En este artículo haremos en una primera etapa un recorrido por la vida de Angus Deaton, desde su Edimburgo natal hasta su distinguida vida académica en Princeton, para poder entender mejor la dimensión de su obra y las circunstancias en las que desarrolló sus teorías. Su biografía nos servirá de marco inmejorable para profundizar posteriormente en los tres ámbitos por los que ha recibido el prestigioso galardón: el desarrollo del Sistema Casi Ideal de Demanda, sus análisis sobre la teoría del consumo intertemporal y sus principales aportaciones a la economía del desarrollo.

\section{Biografía de Angus Deaton: una vida resolviendo paradojas}

«Hay algo muy excitante en realizar una predicción basada en una teoría que no es nada obvia pero que finalmente queda confirmada por los datos». La cita es de Angus Deaton y define a la perfección el atractivo de la economía. Es posiblemente este estímulo el que ha empujado (desde que Adam Smith publicara en 1776: Una investigación sobre la naturaleza y causas de la riqueza de las naciones) a infinidad de intelectuales, tanto economistas como no economistas, a dedicar su vida al estudio de esta ciencia social. Es además un inmejorable resumen de la vida y obra de Angus Deaton.

Nació en Edimburgo en 1945, y, como muchos otros grandes economistas, Angus Deaton encontró su verdadera vocación de forma tardía y casual.
Consciente de la importancia de una buena educación que él nunca tuvo, su padre le envió al prestigioso colegio público Fettes de Edimburgo. Al terminar el bachillerato, Deaton fue admitido en Cambridge para estudiar matemáticas gracias a su habilidad para jugar al rugby. Sin embargo, las aburridas clases de matemáticas le empujaron a la mucho más divertida vida estudiantil de pintas de cerveza y juegos de cartas. Amenazado con la expulsión, sólo había una opción para aquellos estudiantes sin rumbo: la economía. Deaton no quiso decepcionar a su padre, que había hecho un esfuerzo más que notable para proporcionar a su hijo una formación superior, y se vio obligado a realizar el último curso universitario estudiando en la famosa Biblioteca Marshall. Leyendo, escribiendo y debatiendo sobre los Principles of Economics de Samuelson, las teorías del ciclo vital del ahorro de Modigliani y Brumberg, las aportaciones de Hahn y Matthews sobre crecimiento económico, las de Meade sobre comercio o analizando los patrones de consumo de Kuznets, Deaton se enamoró del fascinante mundo de la economía, una ciencia que combinaba análisis teórico, empírico y escritura.

Su último año universitario marcaría fuertemente la carrera profesional de Angus Deaton. Los dos artículos sobre la función de consumo con series de sección cruzada y series temporales de Modigliani y Brumberg le hicieron comprender que un buen marco teórico debe poder explicar toda la evidencia empírica de las series temporales y de las series de sección cruzada, así como los patrones de consumo y renta a nivel internacional. Los trabajos de Kuznets sobre consumo fueron su otra gran influencia y de ellos aprendió la importancia de la medición y de la calidad de los datos. De estas ideas surgirían, años después, sus primeros estudios sobre el consumo que analizaremos en la segunda parte, pero también su incesante búsqueda por perfeccionar las técnicas de medición. Para Deaton, mucho de lo que creemos saber hoy en día sobre el mundo depende de datos que pueden no significar lo que creemos que significan o que simplemente contradicen otros datos a los que, $D$ 
por cuestiones de hábito, damos menor relevancia. Por eso, Deaton se queja amargamente de que en la actualidad los economistas académicos pasan poco tiempo con los productores de datos y de que esto perjudica a ambos colectivos, pues los economistas muchas veces no entienden los datos con los que trabajan y los sistemas de contabilidad nacional se han desarrollado con escasas aportaciones de los usuarios académicos.

Tras finalmente graduarse en 1967 en Cambridge, Deaton tuvo una experiencia agridulce en su primer contacto con el mundo laboral. Había pasado con éxito las exigentes pruebas de acceso del Banco de Inglaterra, pero la institución se encontraba en pleno proceso de transición, sin mucho que ofrecer a jóvenes e inquietos trabajadores recién salidos de la universidad, ávidos por poner en práctica sus conocimientos económicos. Al poco tiempo, decidió aceptar un puesto como ayudante de investigación de su tutor universitario, Jack Revell, en un proyecto de medición de la riqueza nacional. Y, de nuevo de rebote, Deaton encontró su vocación: la investigación académica.

Al poco tiempo de regresar a Cambridge, Deaton conoció e hizo amistad con el posterior premio Nobel Richard Stone, que le incorporó a su equipo para profundizar en el análisis del consumo y la demanda de su "Cambridge Growth Project», un modelo de planificación macroeconómica keynesiano. Los años de colaboración con Richard Stone en el Departamento de Economía Aplicada completaron y enriquecieron la formación de Deaton tanto a nivel profesional como personal. Por aquel entonces, Stone organizaba, al estilo del Grupo de Bloomsbury, multitud de veladas a las que asistían invitados economistas y estadísticos del mundo entero. Deaton y su mujer, Mary Ann, se integraron a la perfección en estos círculos intelectuales de Cambridge. A mediados de los años setenta, Deaton era ya un reputado doctor con varios artículos publicados en la prestigiosa revista Econometrica y un libro escrito sobre sistemas de demanda. Pero más importante todavía, los trabajos con el sistema de gasto lineal de Stone le habían proporcionado una primera línea de investigación económica en forma de paradoja -la paradoja de Deaton- fruto de la cual nacería su hoy famoso Sistema Casi Ideal de Demanda (Almost Ideal Demand System), un trabajo revolucionario considerado uno de los veinte mejores artículos publicados por la American Economic Review en los últimos cien años. La asociación con John Muellbauer -coautor del Sistema Casi Ideal de Demanda- resultó tremendamente fructífera como demuestra que Economics and Consumer Behavior, el libro que escribieron en 1980, se siga vendiendo con un importante número de copias más de 35 años después de su publicación. Unir la economía teórica y la modelización con la experimentación empírica y los datos se convirtió, desde entonces, en uno de los hilos conductores de la obra no sólo de Deaton sino también de una gran parte de la academia.

En 1975, y tras la muerte por cáncer de su mujer Mary Ann, Deaton aceptó dirigir la Cátedra de Econometría en la Universidad de Bristol. Fue durante su estancia en Bristol cuando Deaton se volcó en sus trabajos microeconómicos con Muellbauer, pero durante este tiempo también decidió profundizar sus conocimientos sobre el tratamiento de datos para generar paneles de datos a partir de combinaciones de series temporales y encuestas con datos de sección cruzada.

A principios de los años ochenta, los recortes presupuestarios promovidos por el Gobierno de Thatcher afectaron enormemente a los medios de investigación de la universidad y Deaton decidió en 1983 que era hora de cambiar de aires. Había estado un par de años antes en Princeton como profesor visitante y, cuando en Nueva Jersey se supo de su decisión, no dudaron en ofrecerle un puesto indefinido. Lo suficientemente cerca de Washington y Nueva York como para no estar aislado de la política económica y de las finanzas, pero lo suficientemente lejos como para poder tratar los temas con perspectiva, en la Universidad de Princeton Deaton encontró los medios y el caldo de cultivo necesarios para desarrollar sus ideas. Como reconoció $D$ 
en más de una ocasión, Princeton era todo lo que Deaton había soñado.

El encuentro con Alan Blinder en esta universidad supuso otro punto de inflexión en la carrera de Deaton. Tras escribir un artículo conjunto sobre el ahorro, Deaton comenzó a darle vueltas a las propiedades de las series temporales de consumo y renta. Los principales desarrollos sobre la teoría del consumo intertemporal de Deaton y la crítica de la hipótesis de la renta permanente datan de esta época en la que aprendió que los modelos con agentes representativos podían llevar a engaño y ser irrealistas. El debate continúa, a día de hoy, abierto.

Pese a que había colaborado con el Banco Mundial, antes de llegar a Princeton, en la elaboración de encuestas sobre medición de los niveles de vida, fue en su etapa americana cuando Deaton profundizó en la economía del desarrollo. Arthur Lewis, uno de los pioneros de la materia que se acababa de jubilar en Princeton, y Mark Gersovitz fueron los mentores de Deaton en este campo de la teoría económica, que por aquel entonces se encontraba abandonado frente al auge de las teorías de crecimiento endógeno. Deaton centró sus recursos en intentar mejorar los sistemas de medición de la pobreza y la desigualdad en los países pobres a través del desarrollo de nuevas encuestas de hogares, trabajo que en palabras de Branko Milanovic es todavía la biblia en la economía del desarrollo. Asimismo, junto a Christina Paxson, Deaton aplicó sus conocimientos sobre consumo y renta para explicar fenómenos de desigualdad y realizar sonadas aportaciones sobre las economías de escala de los hogares como The food and household size puzzle.

Precisamente generar enigmas ha sido uno de los principales legados de Deaton como economista. Como él mismo reconocería años después, a la hora de buscar nuevos temas de investigación lo primero que hacía era jugar con los datos hasta que encontraba algo que no lograba entender. Imitando a su padre décadas atrás, cuando tenía que bajar a la mina de un pequeño pueblo de la región de
Yorkshire, Deaton escarbaba en los datos buscando una paradoja, algún dato que no cuadrase con las predicciones de una teoría ampliamente reconocida. La mayoría de las veces, el enigma resultaba ser un simple malentendido, pero cuando encontraba un rompecabezas irresoluble, entonces Deaton encontraba oro. Así surgieron, entre otras, la paradoja de Deaton, la maldición de la abundancia de las materias primas o el enigma de la reducción del consumo de calorías per cápita en India en las últimas dos décadas.

India ha sido una continua fuente de inspiración para Deaton. Encontró en Jean Drèze el perfecto aliado y su trabajo sobre la joya de la corona del antiguo Imperio británico se ha centrado fundamentalmente en la elaboración de índices de precios y en el estudio de cómo éstos afectan a la medición de la pobreza, además de colaborar activamente con el Gobierno indio para mejorar las políticas para combatir la pobreza. Más recientemente ha colaborado con el Programa de Comparación Internacional del Banco Mundial, un consorcio encargado de recopilar millones de precios de bienes de prácticamente todos los países del mundo y de elaborar índices de precios que pueden usarse para comparar las cuentas nacionales de distintos países.

En 2013 Deaton publicó el exitoso libro The Great Escape: health, wealth, and the origins of inequality en donde realizaba un pormenorizado análisis de los efectos de la globalización y del desarrollo de las economías más desfavorecidas. En el prólogo del libro escribió su ya célebre cita: «la existencia es hoy mejor que en prácticamente cualquier otro momento de la historia. Hay más ricos y menos gente sufre pobreza severa. La esperanza de vida es mayor y los padres no deben presenciar cómo se les muere uno de cada cuatro hijos. A pesar de todo, millones de personas aún experimentan los horrores de la indigencia y la muerte prematura. El mundo es enormemente desigual».

También datan de estos últimos años sus críticas a la ayuda oficial al desarrollo. Deaton considera que el desarrollo no es un problema técnico $\triangleright$ 
ni económico sino político, y que la industria de la ayuda al desarrollo, sobre todo en regímenes dictatoriales, impide que los países creen las instituciones necesarias para desarrollarse y cubrir las necesidades de sus ciudadanos. La ayuda al desarrollo desde fuera socorre a muchas personas de países pobres, pero impide en el medio plazo el desarrollo del país.

Más de 30 años después de su llegada, Angus Deaton se ha convertido en un americano de adopción y continúa investigando y dando clases en la cátedra Dwigth D. Eisenhower de Economía y Asuntos Internacionales en la Woodrow Wilson School of Public and International Affairs y en el Departamento de Economía de la Universidad de Princeton, donde trata de llevar junto a su mujer -la también economista Anne C. Case- la idílica vida académica que aprendió de su admirado amigo Richard Stone en Cambridge, hace ya más de 40 años.

\section{Principales aportaciones}

\subsection{El Sistema Casi Ideal de Demanda}

La primera gran aportación por la que Deaton ha sido reconocido, el Sistema Casi Ideal de Demanda, elevó las estimaciones de los sistemas de demanda a un nivel de sofisticación nunca visto hasta entonces. Con la publicación en 1980 de An Almost ldeal Demand System, Angus Deaton y John Muellbauer sentaron las bases de un gran número de desarrollos posteriores, y su aportación se mantiene hasta hoy como una piedra angular en el estudio de la demanda de consumo.

EI Sistema Casi Ideal de Demanda surgió como reacción a las deficiencias observadas en los modelos de demanda anteriores. Desde el siglo XIX, la teoría del consumo convencional se fundamentaba en el proceso de optimización de consumidores individuales racionales, cuya demanda de un determinado bien dependía de los precios de todos los bienes del mercado y del gasto total. El supuesto de racionalidad del consumidor implica que las funciones de demanda cumplen las propiedades de homogeneidad de grado cero en precios y renta, simetría de los efectos sustitución cruzados y negatividad del efecto sustitución precio propio. En 1954, Richard Stone trató de vincular el estudio teórico al análisis empírico por medio del desarrollo del llamado Sistema Lineal de Gasto, que partiendo de una función de utilidad aditiva satisfacía estas propiedades por definición. De esta forma, el modelo no puede ser utilizado para comprobar si la evidencia empírica corrobora la hipótesis de racionalidad del consumidor.

Con el fin de contrastar las propiedades teóricas de la función de demanda, y así comprobar si la evidencia empírica era consistente con el comportamiento optimizador del consumidor, Barten desarrolló entre 1967 y 1969 una versión generalizada del Sistema Lineal de Gasto con datos de la economía holandesa (el llamado modelo de Rotterdam) que le llevó a concluir que estas propiedades podían ser rechazadas.

En 1974, Deaton realizó estimaciones alternativas con datos agregados del Reino Unido y obtuvo resultados similares a los de Barten. No obstante, alegó que estos resultados podían deberse a errores de especificación del modelo empírico, que imponía supuestos demasiado restrictivos sobre el comportamiento del consumidor como para poder corroborar la hipótesis de racionalidad. Otra limitación que Deaton subrayó es que la teoría convencional de la demanda del consumidor estaba formulada a nivel individual, y que aunque las propiedades enunciadas se cumplieran para cada individuo no tendrían por qué hacerlo a nivel agregado. Para hacer frente a estos problemas de agregación, en los años 70 Muelbauer enunció una serie de restricciones mínimas sobre las preferencias que permitían agregar funciones de utilidad individuales. Estas restricciones se cumplen con preferencias de tipo PIGL (PriceIndependent Generalized Linearized) y PIGLOG (Price-Independent Generalized Logarithmic), un caso particular de las primeras con propiedades que las hacen especialmente útiles para el análisis. $\triangleright$ 
A diferencia de las preferencias derivadas de una función de utilidad aditiva, las preferencias de tipo PIGLOG permiten que el gasto en un bien no sea necesariamente proporcional al gasto total del individuo y que las preferencias de los individuos no sean necesariamente idénticas. Además garantizan la existencia de un consumidor representativo que consuma los bienes en las mismas proporciones que la economía a nivel agregado y cuya función de demanda cumple con las propiedades que garantizan la racionalidad de los agentes.

Deaton y Muellbauer utilizaron estas preferencias de tipo PIGLOG para desarrollar el Sistema Casi Ideal de Demanda y con posterioridad estimaron los parámetros del modelo con datos agregados del Reino Unido para comprobar las tres restricciones que imponía la teoría del consumidor racional: homogeneidad, simetría y negatividad del efecto sustitución precio propio. Los resultados obtenidos rechazaron la hipótesis de racionalidad.

No obstante, los propios autores constataron en las conclusiones del estudio y en Economic and Consumer Behavior las debilidades de esta primera versión del Sistema Casi Ideal de Demanda y la necesidad de desarrollos posteriores. Así, consideraban que todo sistema de demanda debía cimentarse en una cuidadosa agregación de consumidores heterogéneos. Por otro lado, reconocieron que el sistema debía incluir otras variables además de los precios y el gasto corriente, como la posible existencia de restricciones del crédito, para ser capaz de explicar los patrones de consumo observados. Estas debilidades impulsaron toda una serie de desarrollos posteriores como el llamado Sistema Casi Ideal de Demanda Cuadrático, más flexible que el original.

Pese a todo, la relativa sencillez, versatilidad y generalidad del sistema de demanda de Deaton y Muellbauer facilitaron su posterior aplicación a campos tan variados como la agricultura, la medición de índices de precios al consumo, la estimación de la desigualdad y la pobreza o las comparaciones internacionales de bienestar. Desde el punto de vista teórico, el Sistema Casi Ideal de Demanda se mantiene aún a día de hoy como una de las herramientas fundamentales para el análisis moderno y la estimación de la demanda.

\subsection{La teoría del consumo intertemporal}

A lo largo de los años ochenta, Deaton se centró en el enfoque dinámico del consumo para estudiar las decisiones intertemporales de consumo y ahorro de los agentes económicos.

El análisis del consumo agregado se había modernizado considerablemente en los años cincuenta y sesenta gracias a las aportaciones de Friedman y Modigliani. Tanto la hipótesis de la renta permanente como el modelo de ciclo vital eran capaces de explicar la evidencia empírica de la suavización del consumo a lo largo del tiempo. En los años setenta, estos modelos eran utilizados extensivamente en la academia. Sin embargo, mientras que éstos se formulaban en términos de elecciones individuales, su evaluación empírica se hacía estudiando los datos de consumo y renta a nivel agregado.

Deaton intuyó que los modelos que partían de un único agente representativo podían colisionar con la lógica de los datos. El modelo de Friedman parte de un programa de optimización intertemporal de un agente representativo, y concluye que éste consumirá una determinada proporción de su renta permanente ${ }^{1}$ en cada periodo, y por tanto de la renta agregada de la economía. Según dicho modelo, la suavidad del consumo en relación a la renta deriva del hecho de que la renta permanente es más estable que la renta disponible en el periodo. Pero Deaton argumentó que la renta agregada per cápita es un proceso estacionario únicamente cuando se toman primeras diferencias y además se trata de un proceso positivamente autocorrelacionado. En definitiva, un incremento no anticipado de la renta, lejos de $\triangle$

\footnotetext{
${ }^{1}$ La renta permanente puede definirse como el flujo de renta que puede ser consumido sin afectar a la fuente de la que procede.
} 
cancelarse debería estar seguido por aumentos adicionales de renta en periodos posteriores. Esto implicaría que la renta permanente es menos estable que la renta corriente. Deaton concluyó que la hipótesis de la renta permanente conducía a predecir que los consumidores aumentarían su consumo presente en una mayor proporción que el aumento de la renta corriente. No obstante, esta predicción no es acorde con la evidencia empírica, que muestra cómo el consumo agregado es menos volátil que la renta agregada. Esta aparente contradicción se conoce desde entonces como la paradoja de Deaton.

La hipótesis de la renta permanente fue reformulada a finales de los años setenta por Robert Hall a través de su hipótesis del paseo aleatorio. En sus aportaciones, Hall parte del programa de optimización intertemporal de un agente representativo con expectativas racionales en un contexto estocástico con mercados de capitales perfectos y demuestra que, bajo ciertos supuestos, el mejor estimador para el consumo futuro es el consumo actual. De esta forma, el cambio en el consumo entre dos periodos no puede predecirse debido a que la decisión de consumo presente incorpora toda la información relevante sobre el resto de variables de la economía. Las variaciones intertemporales del consumo vendrán, por tanto, determinadas por cambios no esperados en las variables, ya que los cambios esperados ya estarán incorporados en las decisiones de consumo actual, esto es, el consumo sigue un paseo aleatorio. En los años ochenta Blinder y Deaton, entre otros, contrastaron empíricamente la hipótesis del paseo aleatorio de Hall y concluyeron que sus resultados podían rechazarse debido a que el consumo mostraba un exceso de sensibilidad ante variaciones previsibles de la renta respecto al comportamiento predicho por esta teoría.

Frente a esta aparente contradicción entre las aportaciones teóricas anteriores y la evidencia empírica, Deaton argumentó que el punto de partida de cualquier teoría del consumo debía ser el estudio de las decisiones individuales de los agentes, cuya renta no siempre fluctúa en el mismo sentido que la renta agregada de la economía. Dado que en un mismo periodo la renta de algunos agentes puede aumentar a la vez que la de otros se reduce, estas variaciones se compensarán a nivel agregado, con lo que la renta agregada presentará una evolución más estable que la renta individual. Esto implica que la variación del consumo agregado, como suma de decisiones de consumo individuales, ante variaciones en la renta individual difiere en gran medida del patrón de consumo de un hipotético agente representativo.

No obstante, el análisis intertemporal de decisiones a nivel individual requería, en principio, la disponibilidad de datos de panel, escasos en el ámbito del consumo. En su lugar, se acometían encuestas periódicas, que proveían datos de sección cruzada. Sin embargo en 1985 Deaton mostró que, si había datos suficientes, entonces se podía construir satisfactoriamente un «pseudo panel» con datos de sección cruzada por grupos de edades uniendo encuestas sucesivas.

Por último, para explicar el exceso de sensibilidad del consumo frente a las variaciones de la renta corriente, Deaton introdujo la existencia de restricciones de liquidez que limitan la capacidad del agente de transformar consumo futuro en consumo presente, en los mercados financieros, para mantenerse sobre su senda de consumo óptima. Por otro lado, la existencia de incertidumbre conduce a los agentes a mantener saldos de ahorro precautorio, de forma que el consumo presenta un exceso de suavidad ante innovaciones informativas con respecto a lo que cabría esperar bajo los supuestos de la hipótesis del paseo aleatorio.

\subsection{Aportaciones a la Economía del Desarrollo}

En los años ochenta la economía del desarrollo constituía un campo de menor rigor teórico que otras áreas de la teoría económica, y se enfrentaba a problemas de escasez de datos para el análisis empírico. Desde entonces, esta rama $\triangleright$ 
ha pasado a contar con una sólida microfundamentación basada en la disponibilidad de datos de elevada calidad gracias, entre otros, al amplio espectro de aportaciones de Angus Deaton en la materia.

Frente al uso de datos agregados de la contabilidad nacional, Deaton defendió la utilización de datos provenientes de encuestas de hogares para medir la pobreza y el nivel de vida de los países. Sus trabajos culminaron en 1997 con la publicación del libro The Analysis of Household Surveys, en donde Deaton, además de explicar el diseño de las encuestas de los hogares y recopilar numerosas series de datos, expuso los principales problemas que surgen cuando se aplican métodos econométricos a las series de datos de encuestas de los hogares, en especial en países pobres.

Asimismo, propugnó que debía ser el consumo, y no la renta, la principal variable en la medición de la pobreza de los países de renta baja debido a la mayor disponibilidad y fiabilidad de datos, así como a su mayor precisión en aquellos casos en los que la renta presenta un mayor componente estacional. No obstante, la pobreza se mide a nivel individual, mientras que los datos sobre consumo se recopilan generalmente a nivel de los hogares, lo que genera el problema de cómo comparar individuos que formen parte de hogares de diferente tamaño y composición. Si se calcula el gasto per cápita de los hogares, y los niños tienen menores necesidades de consumo que los adultos, los hogares con hijos verán sobreestimada su pobreza en términos per cápita. Frente a este problema, Deaton y Muellbauer obtuvieron empíricamente, en 1986, que los niños tendrían que ponderarse como alrededor del 30-40 por 100 de un adulto para obtener una estimación más acertada.

Deaton fue más allá y propuso el concepto de economías de escala de los hogares. Algunos bienes de la economía doméstica, como por ejemplo la calefacción o la propia vivienda, tienen rasgos de bienes públicos, de tal forma que cuanto más grandes sean los hogares (incluso aunque no tuvieran hijos) mayor será su bienestar para un mismo nivel de renta per cápita.
Por otro lado, para medir la pobreza a partir de datos de consumo resulta problemático comparar cifras de distintos países con diferentes precios locales o diferentes calidades de los bienes debido a que los precios de la cesta de bienes de consumo no suelen estar disponibles en los países menos desarrollados. Deaton propuso dividir el gasto por la cantidad consumida para obtener valores unitarios, para después corregirlos por los efectos de calidad y posibles errores de medición y así obtener información sobre precios en los mercados locales.

En los últimos años, la economía del desarrollo ha tratado de dar respuesta a la cuestión de si el crecimiento económico reduce la pobreza de un país. Si el crecimiento generase un aumento de la renta proporcional para toda la población, la respuesta sería inequívoca, pero si éste estuviese repartido de forma desigual la relación de causalidad no sería tan clara. En este campo la evidencia empírica presenta resultados a veces contradictorios. En 2005 Deaton adoptó un enfoque diferente para tratar de explicar estas discordancias basándose en la existencia de divergencias entre fuentes de datos. Así, observó que el consumo agregado medido por las encuestas de los hogares crecía a un ritmo menor que el consumo agregado medido por las cuentas nacionales, y propuso una serie de hipótesis. Por un lado, el consumo de las cuentas nacionales excluye servicios no intercambiados en los mercados, que tienden a ser remplazados por servicios de mercado a medida que el país se desarrolla, provocando una sobreestimación de la tasa de crecimiento del consumo. Por otro lado, el consumo medido por las encuestas de los hogares subestima el consumo medio, y sobreestima la proporción de la población por debajo del umbral de pobreza, debido a que existe una menor probabilidad de que las personas de renta más alta participen en estas encuestas. Finalmente, Deaton concluyó que los procedimientos estadísticos actuales en los países en desarrollo tienden a subestimar la tasa de reducción de la pobreza y a sobreestimar la tasa de crecimiento global. 


\section{Conclusión}

A principios de los años ochenta la teoría económica, la econometría y la economía aplicada eran compartimentos estancos de lo que realmente es una sola materia, la economía. Gracias a economistas como Angus Deaton, la ciencia económica es en 2015 más rica, más completa, más valiosa, más diversa, más polémica, más enriquecedora y más excitante si cabe que hace tres décadas. En definitiva, nos encontramos ante un economista superlativo, de otro tiempo, capaz de integrar fundamentos microeconómicos para ofrecer interpretaciones económicas sobre comportamientos a nivel agregado, y de lograr el difícil equilibrio entre el análisis teórico y empírico, fundamental para la toma de decisiones en política económica. La Real Academia Sueca le ha concedido el prestigioso galardón por sus investigaciones sobre tres áreas estrechamente relacionadas con el consumo, pero el legado de Deaton se extiende por todas y cada una de las ramas de la ciencia económica.

\section{Bibliografía}

[1] BLINDER, A. y DEATON, A. (1985). «The Time Series Consumption Function Revisited", Brookings Papers on Economic Activity, pp. 465521.

[2] DEATON, A. (1974). «The Analysis of Consumer Demand in the United Kingdom», Econometrica, vol. 42.

[3] DEATON, A. (1974). «A Reconsideration of the Empirical Consequences of Additive Preferences», Economic Journal, vol. 84.
[4] DEATON, A. (1975). «The Measurement of Income and Price Elasticities», European Economic Review, vol. 7, pp. 261-274.

[5] DEATON, A. (1981). Essays in the Theory and Measurement of Consumer Behaviour, Cambridge University Press.

[6] DEATON, A. (1981). «The Measurement of Welfare: Theory and Practical Guidelines», The World Bank, LSMS Working Paper $n^{\circ} 7$.

[7] DEATON, A. (1985). «Panel Data from Time Series of Cross- Sections», Journal of Econometrics, pp. 109-126.

[8] DEATON, A. (1991). «Saving and Liquidity Constraints», Econometrica, vol. 59, n 5, pp. 12211248.

[9] DEATON, A. (2005). «Measuring Poverty in a Growing World (or Measuring Growth in a Poor World», Review of Economic Statistics, vol. 87, pp. 1-19.

[10] DEATON, A (2011). «Puzzles and paradoxes: a life in applied economics», Eminent Economists II: Their Life and Work Philosophies. Cambridge University Press.

[11] DEATON, A. (2013). The Great Escape: Health, Wealth and the Origins of Inequality. Princeton: Princeton University Press.

[12] DEATON, A. y MUELLBAUER J. (1980a). «An Almost Ideal Demand System». The American Economic Review, vol. 70, n 3, pp. 312-326.

[13] DEATON, A. y MUELLBAUER J. (1980b). Economic and Consumer Behavior. Cambridge: Cambridge University Press.

[14] DEATON, A. y MUELLBAUER J. (1986). «On Measuring Child Costs in Poor Countries», Journal of Political Economy, pp. 720-744.

[15] DEATON, A. y PAXSON, C. (1998). «Economies of Scale, Household Size, and the Demand for Food», Journal of Political Economy, vol. 106, $\mathrm{n}^{\circ}$ 5, pp. 897-930.

[16] FRIEDMAN, M. (1985). Una teoría de la función de consumo. Alianza Editorial.

[17] HALL, R. (1978). «Stochastic implications of the life cycle-permanent income hypothesis», Journal of Political Economy, vol. 86, pp. 971-987.

[18] ORS, M. (2015). «Mitos de la desigualdad», Actualidad económica, septiembre, pp. 18-26. 
